\section{IL TEATRO COME STRUMENTO FORMATIVO IN CARCERE}

THE THEATRE AS A TRAINING TOOL IN PRISON

Caterina Benelli Università di Messina

\section{Riassunto:}

Abstract:

Il teatro in carcere si è sviluppato negli ultimi The theater in prison has grown over the trent'anni in Italia diventando una delle last thirty years in Italy becoming one of metodologie formative presenti negli istituti the training methods present in prisons. penitenziari. Il contributo intende mettere in The contribution is intended to highlight evidenza l'intervento del teatro in carcere, in the intervention of the theater in prisons, particolare con le donne, tra potenzialità e particularly with women, including potential

Teatro, carcere, donne, pedagogia Theatre, prison, women, prison pedagogy. limiti.

\section{Parole chiave:}

\section{and limits.}

\section{KEY WORD:}

penitenziaria.
Presento questo mio contributo non prima di aver ringraziato Mercedes Arriaga, Eva Moreno e tutto il gruppo di lavoro che ha consentito di realizzare questo seminario di studio interdisciplinare e internazionale. Tale evento mi ha dato la possibilità di riflettere ulteriormente sulla questione del teatro in carcere: una tematica a me cara, poiché mi occupo da quindici anni di pedagogia penitenziaria e di metodologie per l'innovazione dell'educazione formale e non formale negli istituti di pena. Il teatro, in particolare, fa parte delle attività formative più interessanti e maggiormente praticate nell'ambito penitenziario. Sono molti gli istituti penitenziari italiani e internazionali che, ormai da anni, promuovono l'attività teatrale per il trattamento dei detenuti, ma anche per far conoscere la realtà penitenziaria attraverso le produzioni teatrali che portano all'esterno un'altra immagine del soggetto detenuto. In Italia, negli ultimi trent'anni, abbiamo assistito a un importante sviluppo di questa attività non sempre però accompagnato da un'adeguata professionalità tra gli operatori culturali. Non sempre infatti i professionisti che guidano le attività di teatro in carcere possiedono specifiche competenze formative o terapeutiche e ancora troppe poche volte esiste tra loro un confronto interdisciplinare. Ciò determina che le potenzialità dell'attività teatrale si trasformino in elementi di criticità e di rischio per le persone che vivono il periodo della detenzione; si tratta di uomini e donne con problematiche sempre più difficili da gestire all'interno della complessità degli istituti penitenziari.

Prima di affrontare il tema centrale del contributo, ovvero il teatro nel carcere femminile tra opportunità e limiti, vorrei illustrare brevemente la condizione penitenziaria in Italia, con particolare riferimento alla reclusione femminile. All'interno degli istituti penitenziari esiste una questione di genere di cui si parla ancora poco. Secondo i dati statistici del ministero di Giustizia, nel 2015 nei 195 istituti italiani erano presenti oltre 52.000 detenuti a fronte di una potenzialità di circa 49.000 posti con conseguenti problemi di sovraffollamento. Il numero delle detenute negli istituti femminili nel corso degli ultimi dieci anni è sceso da 2.800 nel 2005 a 2.100 nel 2015, con una percentuale che si attesta ormai da qualche anno attorno al $4 \%$ dell'intera popolazione detenuta. Ciò ha significato che le occasioni culturali e formative in carcere siano maggiori negli istituti penitenziari maschili, con una evidente riduzione di proposte educative di tipo formale (attività scolastiche) e non formale (attività culturali professionali e formative di diversa natura) per le donne recluse. Le detenute in Italia sono suddivise in cinque istituti penali e in circa cinquantacinque sezioni femminili e questo divario numerico rispetto alla detenzione maschile determina la mancata progettazione e realizzazione di attività utili al percorso formativo, indispensabile soprattutto negli istituti femminili - per una opportuna progettazione del fine pena e del reinserimento sociale. 


\section{LA SFIDA DELLA FORMAZIONE IN CARCERE: IL RUOLO DEL TEATRO}

Se l'obiettivo della detenzione - come recita l'articolo 27 dell'ordinamento penitenziario italiano - è il trattamento del recluso e della reclusa attraverso un progetto educativo mirato al reinserimento nella società, allora anche il carcere diventa un'occasione di riflessione, di cura, di maggiore consapevolezza di sé e di riprogettazione esistenziale (Benelli, 2008; 2012).

In base a queste considerazioni ci chiediamo: se il carcere dovrebbe essere un luogo di "rieducazione" alla riscoperta di sé, dell'altro e della società, qual è il ruolo del teatro? La risposta sta nella funzione terapeutica e pedagogica dell'attività teatrale che si pone come potenziale agente di miglioramento dei partecipanti e, attraverso la restituzione finale, come ponte tra realtà carceraria e società esterna. La formazione in carcere, dunque, ha l'obiettivo non solo di offrire ai detenuti le possibilità mancate che li avrebbero condotti a compiere altre scelte di vita, ma di facilitare interventi di progettazione educativa passando dalla funzione compensatoria della detenzione alla visione del carcere come ambiente di apprendimento ai fini del reinserimento sociale (Muñoz, 2009; Benelli, Del Gobbo, 2016). Un carcere quindi che, attraverso le occasioni culturali e formative, si pone come "comunità educante" per il miglioramento della condizione attuale di ogni soggetto recluso.

Tra le attività innovative presenti nei progetti pedagogici di istituto, troviamo l'educazione formale (scuola) e non-formale (laboratori di scrittura autobiografica, creativa ed editoriale; laboratori teatrali ed espressivi; corsi professionali). L'attività teatrale, nella fattispecie, ha come oggetto di interesse la persona nella sua formazione ed evoluzione, per promuovere l'espressività del singolo e dei gruppi in favore della realizzazione delle pari opportunità e dell'inclusione sociale.

Ripercorrendo la genesi del teatro in carcere, rintracciamo le prime sperimentazioni negli anni ' 80 quando, fin da subito, i promotori di questa attività ne intuirono le potenzialità ponendo al centro la pratica teatrale piuttosto che lo spettacolo. Il 5 luglio 1982 rappresenta una data storica per il teatro penitenziario: per la prima volta sei detenuti-attori dell'ex Teatro del carcere di Rebibbia a Roma si esibirono al di fuori dell'istituto per iniziativa di un educatore penitenziario che aveva colto le potenzialità dell'attività teatrale in carcere come dispositivo trattamentale. Lo spettacolo Sorveglianza speciale di Jean Genet venne rappresentato all'interno della Rocca di Albronoz a Spoleto: un'esperienza resa possibile grazie alla collaborazione tra il direttore e il gruppo educativo della casa di reclusione con il magistrato di sorveglianza, che concesse permessi eccezionali agli attori, interpretando in maniera estensiva l'articolo 30 dell'ordinamento penitenziario. In quegli anni nacquero compagnie teatrali che ancora oggi calcano la scena del teatro civile e sociale nazionale e internazionale. Nel
1984 la casa circondariale di Brescia nel carcere di San Vittore crea un laboratorio di teatro. A Rebibbia, il 22 dicembre 1986 cinquantatre detenuti del Teatro-Gruppo usufruiscono dei permessi premio introdotti dall'appena approvata Legge Gozzini per le rappresentazioni teatrali. A Volterra, nel 1988 Armando Punzo fonda la Compagnia della Fortezza con la quale precisa metodologie e scopi dell'attività di ricerca teatrale con i detenuti.

Dagli anni' 80 le esperienze di teatro in carcere si sono moltiplicate, l'amministrazione penitenziaria ha apertonuovi spazi, sostenuto progettidisperimentazione e formazione, promosso la collaborazione con gli enti locali e culturali. Alcune esperienze hanno assunto una dimensione europea e, dal 2014, il 27 marzo si festeggia ogni anno la Giornata nazionale del teatro in carcere, in concomitanza con la Giornata mondiale del teatro indetta dall'Istituto internazionale del teatro presso la sede Unesco di Parigi.

Oggi le compagnie teatrali che lavorano negli istituti penitenziari sono più di cento: sono gruppi e compagnie che, pur con differenti caratteristiche operative e stilistiche, realizzano spettacoli in cui la qualità espressiva e artistica si connette (o dovrebbe connettersi) ai fini pedagogici della pratica teatrale. Molti gruppi si esibiscono anche nei teatri cittadini, sviluppando così una sensibilizzazione sociale alla promozione dell'incontro per il contrasto al pregiudizio nella comunità stessa. In altri casi invece, sono stati $\mathrm{i}$ teatri interni alle carceri ad aprire le porte al pubblico creando un ponte tra "dentro" e "fuori" le mura. Nell'istituto romano di Rebibbia, ad esempio, operano tre compagnie teatrali costituite dall'associazione "La ribalta - Centro studi Enrico Maria Salerno" che ha collaborato con i fratelli Taviani nella realizzazione del film Cesare deve morire, vincitore dell'Orso d'oro al $62^{\circ}$ Festival di Berlino e di cinque David di Donatello nel 2012

L'azione formativa del teatro risiede soprattutto nel processo di promozione e di emancipazione del soggetto detenuto. Come già sottolineato, infatti, l'aspetto centrale dell'attività teatrale sta nel percorso formativo e non nella rappresentazione finale che, comunque, funziona da rinforzo e da restituzione al gruppo, all'istituto e al territorio.

Il prolungarsi nel tempo di importanti progetti teatrali nelle carceri italiane e l'emergere di nuove proposte legate a nuove compagnie teatrali hanno sviluppato un tessuto di esperienze diversificate fra loro: esperienze condotte da uomini e donne del teatro professionale italiano, che hanno offerto il loro contributo al teatro nelle carceri italiane (e non solo). Questi percorsi hanno consentito di creare progressivamente metodi d'intervento progettati e gestiti con stili e linguaggi inediti che richiedono di essere sviluppati e studiati a livello interdisciplinare, in particolare dalle scienze della formazione e psicologiche per modellizzare e valorizzare tecniche e strumenti terapeutici ed educativi nell'attività teatrale in carcere. 


\subsection{Il caso di Volterra}

Tra le circa cento compagnie e associazioni teatrali italiane, la Compagnia della Fortezza di Volterra ${ }^{1}$ è sicuramente tra quelle più interessanti sul panorama internazionale. Essa si avvale della collaborazione dell'associazione "Carte Blanche", impegnata a tutelare e valorizzare il lavoro svolto sia all' interno della casa di reclusione La Fortezza di Volterra che all'esterno attraverso tournée. Grazie all'instancabile lavoro del suo direttore e ideatore, Armando Punzo, e di altri professionisti, la Compagnia della Fortezza porta avanti dal 1988 un lavoro trattamentale riconosciuto a livello internazionale.

Durante l'anno, insegnanti, educatori, psicologi e operatori teatrali collaborano in modo interdisciplinare per la realizzazione del progetto finale. È nato a Volterra qualcosa di nuovo e di completamente originale: un tipo di teatro fondato sull'ascolto dei luoghi in cui opera, sulle biografie delle persone coinvolte, sulla reinvenzione continua dei linguaggi scenici secondo i limiti imposti dalle strutture e dalle condizioni eccezionali di questa particolare forma di lavoro teatrale. Limiti che spesso sono diventati occasioni e opportunità. Assistiamo a forme teatrali in cui si intrecciano fortemente sperimentazione e tradizione scenica italiana ed europea. Un teatro che privilegia la scrittura scenica sia quando essa affronta testi o autori classici della cultura europea (da Don Chisciotte ad Alice, da Pinocchio a Shakespeare, da Genet a Eduardo De Filippo), sia quando procede attraverso forme di autodrammaturgia. Nelle carceri italiane si sono sviluppate forme di scrittura scenica fra loro differenziate: dalle case circondariali (che ospitano detenuti in attesa di giudizio o di trasferimento) alle case di reclusione, dalle carceri femminili a quelle minorili fino alle strutture psichiatriche, si cerca di coniugare l'utilità che queste esperienze laboratoriali e produttive hanno per i detenuti con la creazione di un teatro di evidente valenza artistica e comunicativa. Come dicevamo, il teatro in carcere è un'attività laboratoriale ricca di ricadute sociali anche attraverso la possibilità di ospitare spettatori nelle strutture carcerarie, oppure di rappresentare nei teatri ufficiali gli spettacoli prodotti in carcere per una promozione del teatro come ponte tra carcere e territorio. Il teatro di Volterra si inserisce in questo quadro andando oltre la prosa e utilizzando linguaggi nei quali le culture e le lingue possono incrociarsi per creare nuove alchimie sceniche. In questo senso il teatro in carcere appare come un'esperienza teatrale popolare e di elevata qualità artistica.

\section{IL TEATRO NEL CARCERE FEMMINILE TRA POTENZIALITÀ E LIMITI}

La questione femminile in carcere rende ancora più complessa la già difficile situazione della detenzione in Italia e richiede una maggiore attenzione nella gestione

1 Per approfondimenti si consulti il sito: http://www.compagniadellafortezza.org/new/carte-blanche/ carte-blanche/ e nella realizzazione degli interventi formativi. Infatti, la condizione detentiva delle donne

molto articolata: a partire dalla relazione interrotta con i figli, passando dai disagi psichiatrici fino alla tossicodipendenza, essa ci pone questioni che determinano risposte più specifiche e multiformi. Una recente ricerca sulla condizione femminile didetenzione (Ronconi, Zuffa, 2015) ${ }^{2}$, finalizzata a prevenire l'autolesionismo e il suicidio nelle carceri femminili e realizzata attraverso un modello di intervento preventivo, di contenimento della sofferenza e di promozione della salute in carcere, dimostra l'importanza di attività formative mirate alla gestione del disagio psicologico, offrendo alle donne la possibilità di ripensarsi e confrontarsi con se stesse e con il gruppo di riferimento.

La donna detenuta è una donna fragile nella costruzione dell'identità personale e di genere e ha bisogno di essere accompagnata verso una riorganizzazione della propria vicenda esistenziale, tramite l'organizzazione di iniziative per gestire il tempo libero all'insegna della revisione e della riprogettazione del sé, in vista del fine pena e del reinserimento sociale.

Tra le attività proposte nelle sezioni femminili degli istituti penitenziari, come già abbiamo detto, il teatro è uno dei pochi percorsi laboratoriali presenti, considerando anche l'importante riduzione dei finanziamenti da parte degli enti locali per attività culturali e formative in carcere. Nonostante ciò, in Italia tra le varie proposte teatrali presenti, meritano di essere citati i casi di Bollate (Milano) e di Rebibbia (Roma), nelle cui sezioni femminili da alcuni anni si sono sviluppate attività teatrali di forte impatto formativo a livello individuale e sociale. ${ }^{3}$

Gli istituti penitenziari del territorio nazionale in questo periodo storico sono caratterizzati da specifiche difficoltà di ordine politico e sociale, quali: sovraffollamento; carenza di personale; orientamenti e decisioni politiche che fanno temere un ritorno a un carcere non rieducativo ma prevalentemente esecutivo della pena; presenza sempre maggiore di detenuti non italiani con evidenti problemi di povertà, difficoltà comunicative, linguistiche e relazionali; presenza significativa di giovani che, per piccoli

2 Recluse. Lo sguardo della differenza femminile sul carcere è una ricerca-azione con l'obiettivo di dare voce alle donne invisibili: le recluse. Dalla ricerca e attraverso le voci delle protagoniste, emergono le seguenti questioni: storie di sopravvivenza e di resistenza; un corpo violato; processo di infantilizzazione; deprivazione affettiva (madri interrotte); solidarietà tra donne: strategia di coping; strategie per gestire l'ansia (musica, scrittura, lettura, attività espressive). Un'interessante esperienza di "sopravvivenza affettiva" in carcere è data dalla presenza del panneggio: un modello comunicativo che serve per conversare da una finestra all'altra degli istituti penitenziari attraverso un vocabolario interno e che si tramanda in carcere.

3 Per il carcere femminile di Milano-Bollate segnaliamo la compagnia del Teatro In-Stabile che opera dal 2008 presso il teatro interno della casa di reclusione, mentre per il carcere femminile di Rebibbia segnaliamo la Compagnia delle Donne del Muro Alto attiva dal 2013. 
reati, riempiono gli istituti penitenziari dove assorbono o rafforzano comportamenti devianti e delinquenziali.

In questa situazione, progettare e praticare il teatro in carcere, così come le altre attività formative, appare sempre più difficile. È stato perciò creato il “Coordinamento nazionale Teatro in Carcere", sorto all'interno delle carceri italiane in questi decenni e capace di offrire progettazione, relazione, luoghi di confronto e di qualificazione del movimento teatrale. Nonostante le difficoltà, molti sono i contesti penitenziari dove si realizzano le attività teatrali, le cui potenzialità, in particolare nelle sezioni femminili, sono riconducibili alla sua funzione terapeutica e pedagogica come potenziale agente di cambiamento e miglioramento, al suo ruolo di ponte tra mondo carcerario e società esterna, attraverso l'incontro tra differenti storie, e in quanto strumento di contrasto alle forme di pregiudizio tramite la "restituzione" teatrale alla comunità.

In riferimento alle metodologie efficaci per la formazione teatrale in carcere, sottolineiamo i seguenti aspetti:

-lo studio del testo e l'esercizio mnemonico come rinforzo di competenze linguistiche e di miglioramento di capacità narrative;

-l'esercizio di scrittura di sé come opportunità riflessiva e di rispecchiamento nell'altro;

-l'esperienza relazionale e i nuovi sguardi su se stessi e sugli altri attraverso un processo empatico;

-la possibilità di ascoltarsi, di ripensarsi, di aprirsi ad altre storie e altre possibilità per "evadere" dalla ripetitività della quotidianità carceraria;

-la possibilità di migliorare le relazioni in carcere tra i detenuti stessi e tra i detenuti e il personale (educatori, psicologi, polizia penitenziaria).

Il teatro in carcere apre alla comunicazione attraverso un linguaggio nuovo rispetto a quello deviante conosciuto dalla popolazione detenuta e permette di narrare altre storie, di conoscere mondi diversi e comprendere gli altri con atteggiamento non giudicante. Può inoltre essere uno strumento utile per il futuro professionale, come è accaduto per alcuni ex detenuti diventati attori e operatori culturali.

È fondamentale evidenziare però che proporre il teatro in carcere può avere anche ricadute negative se non affrontato con cura e con le competenze pedagogiche e terapeutiche necessarie alla gestione di situazioni sempre più complesse, presenti in particolare nelle sezioni femminili degli istituti penitenziari.

Il teatro in carcere diventa terapeutico solo se realizzato da professionisti competenti e da èquipe interdisciplinari che comprendano figure diverse per la gestione delle multiproblematiche che emergono o potrebbero emergere durante e dopo l'attività.
Penso alle varie figure di operatori presenti in carcere (educatori, insegnanti, volontari) che prestano servizi diversi e che potrebbero collaborare anche a favore dei laboratori teatrali valorizzando, contemporaneamente, le proprie attività formative e culturali.

L'attività teatrale in carcere, oltre a offrire molteplici occasioni formative, richiede quindi attenzioni particolari e competenze non solo artistiche e teatrali, ma anche educative e/o terapeutiche per la gestione delle dinamiche individuali e di gruppo che scaturiscono, immancabilmente, dall'attività stessa. Sovente le detenute portano in carcere situazioni difficili quali: disturbi psichici, problemi di dipendenza da sostanze psicoattive, vissuti di prostituzione e di violenza. Si tratta di sofferenze che rischiano di generare situazioni di autolesionismo e di aggressività verso gli altri nelle stesse sezioni del carcere se non gestite adeguatamente. Proporre attività formative, mirate alle specifiche tipologie di personalità e di disagio delle donne recluse, diventa occasione per facilitare un percorso terapeutico adeguato ai loro bisogni. La direzione dell'istituto penitenziario, per questi motivi, ha il compito di selezionare, proporre e supervisionare le opportunità formative che sono presentate dall'amministrazione comunale del territorio di competenza, dalle associazioni culturali e di volontariato e da singoli esperti che mettono a disposizione le proprie competenze a favore $\mathrm{di}$ attività culturali in carcere. Non sempre la direzione e gli educatori penitenziari hanno la possibilità di seguire e monitorare le attività che vengono proposte nell'istituto penitenziario; spesso invece sono gli stessi operatori culturali che propongono l'attività teatrale senza coordinarsi con l'area educativa dell'istituto. La mancanza d coordinamento e di comunicazione tra personale esterno e interno genera il rischio di operare avendo in vista solo lo spettacolo finale e l'aspetto artistico dell'attività culturale, a scapito del processo formativo, riflessivo e trattamentale che, come già detto, è la vera occasione di miglioramento e di cambiamento per le persone, in particolare per le donne recluse. Progettare attività formative attraverso il lavoro di un'équipe interdisciplinare consente a ogni professionista di offrire un contributo specialistico attraverso il confronto finalizzato alla realizzazione di un'azione formativa e di un progetto educativo comune, coerente e mirato al lavoro individuale e di gruppo. La questione del lavoro di rete - accanto alle competenze sempre più specifiche richieste - è, a mio parere, la sfida della formazione in carcere in questo periodo storico.

Concludo con le parole di Cosimo Rega, protagonista del film Cesare deve morire, che con più forza ci aiutano a comprendere la condizione della reclusione e il bisogno di opportunità per ripensarsi e riprogettarsi provato dai detenuti:

Non mi bastava sopravvivere al carcere. Volevo vivere. Per questo ho fatto teatro Perché un detenuto è prima di tutto un uomo, con le sue emozioni, le sue paure. E i suoi dolori. Siamo persone che hanno il diritto e il dovere di riscattarsi, di dimostrare, al di là di quello che abbiamo fatto, tutta la nostra umanità. 


\section{RIFERIMENTI BIBLIOGRAFICI}

Benelli, C. y Del Gobbo, G., Lib(e)ri di formarsi. Educazione degli adulti non formale in carcere, Pacini, Pisa, 2016.

Benelli, C., Promuovere formazione in carcere, Del Cerro, Pisa, 2008.

----, Coltivare percorsi formativi. La sfida dell'emancipazione in carcere, Liguori, Napoli, 2012.

----, “Nascita e sviluppo di un'idea: i laboratori autobiografici in carcere", in Mnemosyne: Vitesse ou lenteur dans récits autobiographiques su la naissance des idées, Presses Universitaires de Louvain, n. 92016.

Burón, Nistal, Javier, "El futuro de la cárcel y la cárcel del futuro", in Marci, Salvatore, “Teatro e carcere: lo sguardo negato", Rivista on-line, Ristretti Orizzonti.

Mancini, A., A scene chiuse. Esperienze di teatro in carcere nel mondo, Titivillus, Pisa, 2008.

Muñoz, V., Promotion and protection of human rights, civil, political, economic, social and cultural rights, including the right to development. The right to education of persons in detention, A/HRC/11/8, GE.09--12758 (E) 230409, 2009.

Pereira, C., "Cine, cárcel y mujeres. Un ejemplo de creación de conocimiento", Revista Venezolana de Información, Tecnologia y Conocimiento, N. 2, 2009.

Ronconi, S., Zuffa, G., Recluse. Lo sguardo della differenza femminile sul carcere, Ediesse, Roma, 2015.

Citazioni da internet:

http://www.compagniadellafortezza.org/new/carte-blanche/carte-blanche/. 